\title{
IMPACT OF AGE ON OUTCOME OF COVID-19 PATIENTS AT A TERTIARY CARE HOSPITAL
}

\author{
Iffat Rafique, Muhammad Yasir, Nadeem Zia, Irfan Najm Sheen, Hamid Jamal Siddiqui, Asif Nadeem \\ Combined Military Hospital Malir/National University of Medical Sciences (NUMS) Pakistan
}

\begin{abstract}
Objective: To assess the impact of age on outcome of COVID-19 patients at a tertiary care hospital. Study Design: Cross sectional study.

Place and Duration of Study: Combined Military Hospital Malir, Karachi, from Apr to Jun 2020.

Methodology: This was a retrospective cross sectional study conducted at Combined Military Hospital Malir Karachi. About 120 positive cases of SARS COV-2 were studied, including males and females. Age range was 21-85 years. Patients were divided into groups according to age, group A (21-40 years), group B (41-55 years), group C (56-85 years). Presenting complaints and associated co morbidities were also analyzed. Mild cases were managed conservatively. Moderate to severe oxygen dependent cases were managed in intensive care units.

Results: The mean age of patients was 40.24 years with $16.25 \pm$ SD. Co- morbidities were noted in 67 (55.83\%) patients, highest in group C, hypertension being the most common. Ten patients could not survive, due respiratory failure complicated by adult respiratory distress syndrome (ARDS). Bilateral infiltrates $>50 \%$ observed in $(14 \%)$ out of ( $n=120)$ patients. One hundred and ten patients were discharged with uneventful recovery.

Conclusion: Mortality was highest among elderly patients with comorbidities. Therefore, international standing operative measures (SOPs) to be followed. Meticulous monitoring of vital signs and oxygen saturation in mild cases is the bare minimum requirement and timely appropriate treatment escalation of moderate to severe cases in intensive care units is the key to success.
\end{abstract}

Keywords: Age, Coronavirus infection, Comorbidities, Outcome.

This is an Open Access article distributed under the terms of the Creative Commons Attribution License (https://creativecommons.org/licenses/by-nc/4.0/), which permits unrestricted use, distribution, and reproduction in any medium, provided the original work is properly cited.

\section{INTRODUCTION}

COVID-19 is a rapidly evolving pandemic disease, emerged in December 2019 at Wuhan City, Hubei Province of Central China. Since then, a series of pneumonia cases reportedwith history of exposure to the food at Huanan wild animal market. Later, numbers of cases noted to became positive even without eating the particular seafood, therefore contributes to the possibility of human to human transmission. On January 7, 2020, Chinese experts named the acute respiratory syndrome as novel coronavirus 2019 (SARS-CoV-2). On January 30, World Health Organization declared the outbreak as global pandemic and renamed the disease as coronavirus disease 2019 (COVID-19) ${ }^{1}$. According to the World Health Organization (WHO), about 216 countries have been affected worldwide, there have been $54,301,156$ confirmed cases of COVID-19, including $1,316,994$ deaths $^{2}$.

In Pakistan, first two cases were reported through the Federal Ministry of Health Islamabad on February 26th 2020, at Karachi and Islamabad3. In 2002, Severe Acute Respiratory Syndrome Coronavirus (SARS-CoV) pandemic erupted at China, with the mortality up to

Correspondence: Dr Iffat Rafique, Classified Medical Specialist, Combined Military Hospital Malir Pakistan

Received: 11 Sep 2020; revised received: 30 Nov 2020; accepted: 17 Dec 2020
11\%. In 2012, Middle East Respiratory Syndrome Coronavirus (MERS-CoV) emerged at Saudi Arabia, with a mortality of $37 \%$. Both originated from bats and transmitted in human beings through intermediate hosts, the civet (Paguma larvata) and camel respectively 4

Coronavirus disease affected all ages groups, however elderly population, with underlying co-morbidities, were highly vulnerable to acquire infection and poor outcome ${ }^{5}$. Symptomsvaries from mild upper respiratory tract illness to severe pneumonia, respiratory failure and death ${ }^{6}$. Internationally, mortality was noted to be highest among older individuals, with 80 percent of deaths occurring in those aged $\geq 65$ years. Out of those, $>75 \%$ were suffering from co-morbidities as ischemic heart disease, diabetes mellitus, hypertension, chronic obstructive airways disease, malignancies, chronic kidney disease, and epilepsy ${ }^{7,8}$.

With advancing age, immune mechanism losses its resiliency, could increases the susceptibility to infections. Therefore, frequent hand washing, avoiding unnecessary travelling, limited outdoor gatherings can prevent the spread of second wave of the pandemic ${ }^{9}$.

\section{METHODOLOGY}

This cross sectional study was conducted at department of Medicine, Combined Military Hospital Malir Karachi, from April to June 2020. Data collection 
was done with the permission of Hospital Ethical Committee (ref letter no. 05/2020/Trg. Adm). Informed consent obtained from all the participating patients. One hundred and twenty indoor patients, males and females were included in the study. All cases were laboratory confirmed PCR (polymerase chain reaction) positive for SARS-COV-2. Children and outdoor cases were excluded from the study. Sampling strategy was non-probability convenient technique. Patients were divided into 3 age groups; group A 21-40 years, group B 41-55 years, group C 56-85 years.

Mild cases, which do not require oxygen supplementation, were managed in wards with symptomatic treatment. Moderate to severe cases, including those who were oxygen dependent were managed in intensive care units, with injectable dexamethasone, to cilizumab, carabapenem, plasma exchange and assisted ventilation according to severity of cases. Investigations including complete blood picture, liver function tests, renal function tests, serum ferritin, C-reactive proteins, $\mathrm{D}$ dimers and lactate dehydrogenase, arterial blood gases, chest radiographs and high resolution computed tomography (HRCT) chest of selected cases were performed. Data was analyzed using SPSS-24. Descriptive analysis with mean \pm standard deviation was calculated for age. Co-morbiditiesand presenting complaints were analyzed as qualitative variables.

\section{RESULTS}

A total of 120 patients were included in the study. Male patients were $92(76.7 \%)$ and females were 28 (23.3\%). The mean age of patients was 40.24 years with $16.25 \pm$ SD. Age range from 21-85 years. They were divided into 3 groups; $21-40$ years (group A), 4155 years (group B), 56-80 years (group C) (fig-1). Comorbidities were noted in $67(55.83 \%)$ patients, mostly observed among group C. Hypertension was the most commonly noticed comorbidity followed by diabetes mellitus, bronchial asthma and others (fig-2).

Ten $(8.3 \%)$ patients died, 3 were female and 7 males. One patient from group A was a young female with no known co morbidities unfortunately entered CRS (cytokine release syndrome) with fatal complications, 3 patients from group B, and 6 patients from group $C$. Four were placed on noninvasive mechanical ventilation, none could be survived due secondary complications as adult respiratory distress syndrome (ARDS). Bilateral infiltrates $>50 \%$ on chest radiograph were observed in $(14 \%)$ of the $(n=120)$ patients. One hundred and ten patients were discharged with complete recovery.

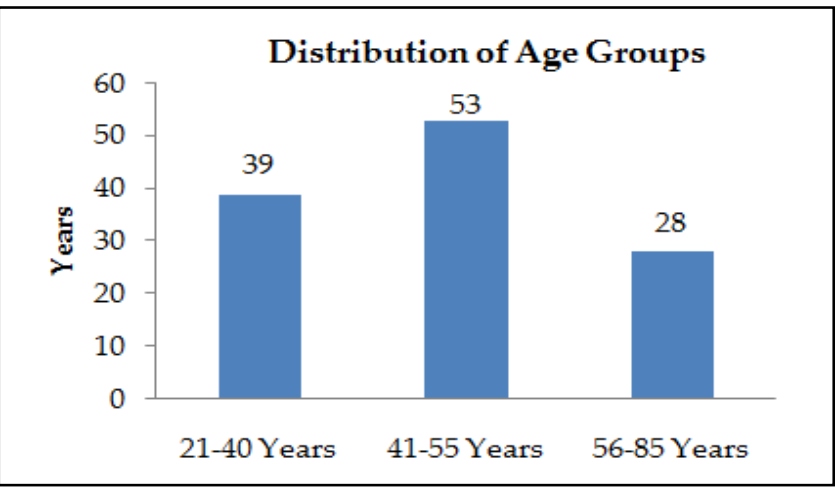

Figure-1: Distribution of study group according to age.

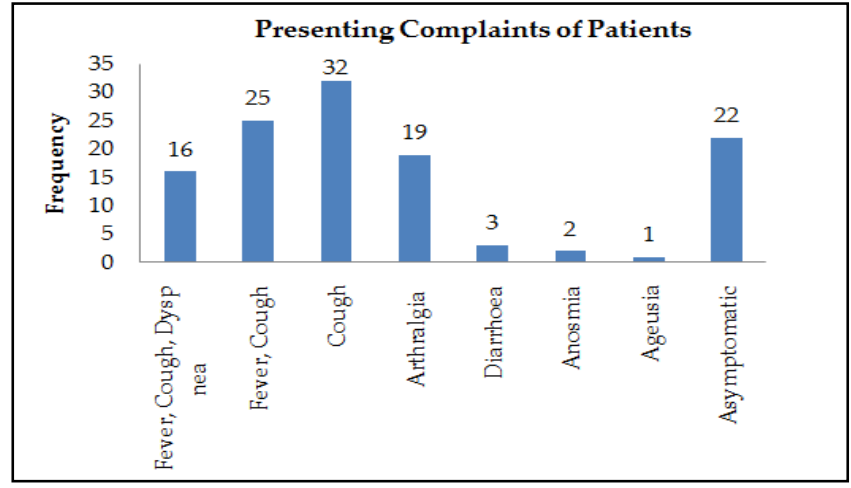

Figure-2: Presenting complaints in study group.

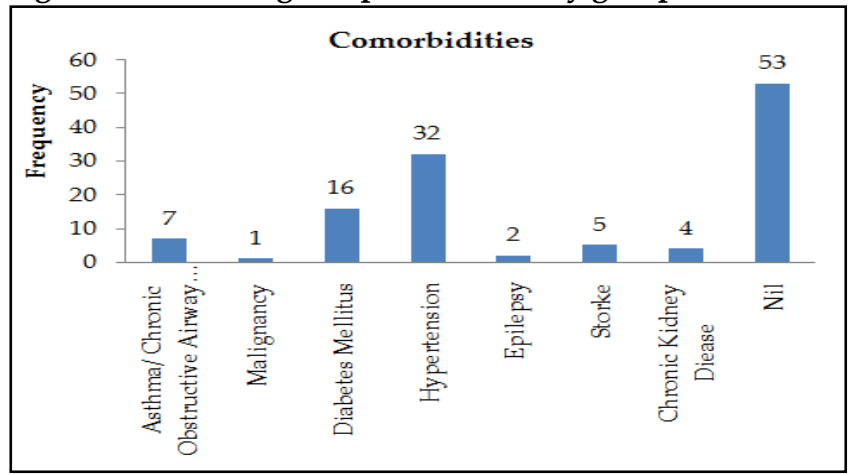

Figure-3: Co morbidities noted in PCR positive cases.

\section{DISCUSSION}

Globally, the coronavirus cases surpass previous pandemics and have been keep on increasing during the second surge worldwide ${ }^{10}$. Considering the source of host-to-human transmission Hunan seafood market has been totally banned for trade purposes ${ }^{11}$. Chinese data revealed that elderly people, with co-morbidities were prone to acquire coronavirus infection as compared to the healthy individuals ${ }^{12}$. According to a report from the Chinese Centre for Disease Control and Prevention, indoor patients with moderate to severe disease had mean age ranged from $49-56$ years ${ }^{13}$. That coincides with our study where the mean age was 40.24 years with $16.25 \pm \mathrm{SD}$. In Europe, the case fatality 
rates were 8 and 15\% among those aged 74-80 years or older, respectively, in contrast to the $2.3 \%$ case fatality rate among the healthy population. InItaly, case fatality rates were 12 and $20 \%$ among those aged 74-80 years or older, respectively ${ }^{14}$.

In the United States of America, mortality was highest among older individuals admitted in intensive care units, with $80 \%$ of deaths occurring in those aged $\geq 65$ years, $>80 \%$ of those were suffering from co-morbidities including ischemic heart disease, diabetes mellitus, hypertension, chronic obstructive airways disease, malignancies, chronic kidney disease, and obesity 15. Data from various countries supports the evidence that advanced age and comorbidities are independent predictors of in-hospital mortality for COVID-19 patients ${ }^{16}$.

It has been hypothesized that due advanced age dependent defect in T-cell and B-cell function, moreover the increased production of type 2 cytokines could lead to a deficiency in control of viral replication and prolonged pro inflammatory response, leading to poor outcome ${ }^{17}$. Even in rural areas of US, and UK, it has been observed that PCR positive critically ill hospitalized patients with co morbidities on supplemental oxygen had poor outcome. According to a report by Georgia Department of Public Healthin March 2020, an interesting finding noticed that female gender is an independent predictor of reduced mortality, probably because they usually remains indoor therefore less prone to acquire the droplet infections ${ }^{18}$. In an analysis of nearly 300,000 confirmed cases with moderate to severe disease in the United States tertiary care hospital, the mortality rate was 12 times higher, among patients with co-morbidities compared with none ${ }^{19}$.

We noticed in our study that males have a higher mortality rate as compared to females 7:3. All over the globe including China, Spain, Italy, Germany, United States, India and Pakistan it has been noticed that male population were severely affected irrespective of social disparities ${ }^{20}$. Reason being, males have outdoor exposure at markets, workplaces and institutes, thus even the precautionary measures may not be sufficient to prevent the spread of disease.

Despite all the advanced treatment protocols, life threatening complications have been observed in number of critical cases. Fatal cases in this study group suff-ered from acute respiratory distress syndrome (ARDS), hospital acquired pneumonia, cardiomyopathy, acute liver and kidney injury, and multiorgan dysfunction ${ }^{21}$.

\section{CONCLUSION}

Round the globe, mortality was highest among elderly patients with comorbidities. Therefore, international standing operative measures (SOPs) to be followed. Meticulous monitoring of vital signs and oxygen saturation in mild cases, and timely appropriate treatment escalation of moderate to severe cases in intensive care units is the key to success. Multi-diciplinary approach offers best option for the improvement of final outcome.

\section{CONFLICT OF INTEREST}

This study has no conflict of interest to be declared by any author.

\section{REFERENCES}

1. Zhu N, Zhang D, Wang W, Li X, Yang B, Song J, et al. A novel coronavirus from patients with pneumonia in China, 2019. New Engl J Med 2020; 382(8): 727-33.

2. Bilgin S, Kurtkulagi O, Kahveci GB, Duman TT. Millennium pandemic: a review of coronavirus disease (COVID-19). Exp Biomed Res 2020; 3(2): 117-25.

3. Zhou P, Yang XL, Wang XG, Hu B, Zhang L, Zhang W, Chen HD. A pneumonia outbreak associated with a new coronavirus of probable bat origin. Nature 2020; 579(7798): 270-73.

4. COVID-19 live dashboard (Pakistan): national institute of health Islamabad. [Internet] https://www.nih.org.pk/novel-coranavirus-2019-ncov. (assessed at: 20 Apr 2020)

5. McMicheal TC, Clark S, Pogosjans S, Kay M, Lewis J, Baer A, et al. COVID-19 in a Long-Term Care Facility- King County, Washington, March 9, 2020. MMWR Morb Mortal Wkly Rep 2020; 69(12): 339-42.

6. Sanya ZC, Zhong DR. Clinical Characteristics of COVID-19 in China. N Eng J Med 2020; 382: 1708-20.

7. Wang LS, Wang YR, Ye DW, Liu QQ. A review of the 2019 Novel Coronavirus (COVID-19) based on current evidence. Int J Antimicrob Agents 2020; 55(6): 105948

8. National action plan for preparedness \& response to Corona virus disease (COVID-19) Pakistan. https//www.nih.org.pk/ wp-content/uploads/2020/02/NAP-covid-19_AL@version-3date-12-2-2020-with-annexures.pdf

9. 9.Tsang KW, Ho PL, Ooi GC, Yee WK, Wang T, Yeung MC, et al. A cluster of cases of severe acute respiratory syndrome in Hong Kong. N Engl J Med 2003; 348(20): 1977-85.

10. Li Q, Guan X, Wu P, Wang X, Zhou L, Tong Y. Early transmission dynamics in Wuhan, China, of novel coronavirus-infected pneumonia. N Engl J Med 2020; 382: 1199-207.

11. Man Ha K. A lesson learned from the MERS Outbreak in South Korea in 2015. J Hosp Infect 2016; 92(3): 232-34.

12. Wong JEL, Leo YS, Tan CC. COVID-19 in Singapore - current experience: Critical global issues that require attention and action. J Am Med Assoc 2020; 323(13): 1243-44.

13. Xu X, Chen P, Wang J, Feng J, Zhou H, Li X, et al. Evolution of the novel coronavirus from the ongoing Wuhan outbreak and modeling of its spike protein for risk of human transmission. Sci China Life Sci 2020; 63(3): 457-60.

14. Imam Z, Odish F, Gill I, Armstrong J, Vanood A. Older age and comorbidity are independent mortality predictors in a large cohort of 1305 COVID-19 patients in Michigan, United States. J Inter Med 2020; 288(4): 469-76. 
15. Lee N, Hui D, Wu A, Cameron P, Joyut GM, Ahuja A, et al. A major outbreak of severe acute respiratory syndrome in Hong Kong. N Eng J Med 2003; 348(20): 1986-94.

16. Richardson S, Hirsch JS, Narasimhan M, Crawford JM, Mc Ginn T, Davidson KW. Presenting characteristics, comorbidities, and outcomes among 5700 Patients Hospitalized with COVID-19 in the New York City Area. J Am Med Assoc 2020; 323(20): 2052-59.

17. Feng Y, Ling Y, Bai Y, Xie Y, Huang J, Li J, et al. COVID-19 with different severities: a multicenter study of clinical features. Am J Respir Crit Care Med 2020; 201(11): 1380-88.

18. Centers for disease control and prevention. 2019 Novel coronavirus, Wuhan, China. Information for Healthcare Professionals.
[Internet] https://www.cdc.gov/coronavirus/2019-ncov/hcp/ index.html (Assessed at: Apr 20, 2020))

19. Huilan $\mathrm{Tu}$, Sheng $\mathrm{Tu}$, Anwen S, Jifang S. Current epidemiological and clinical features of COVID-19; a global perspective from China. J Infect 2020; 81(1): 1-9.

20. Wu C, Chen X, Cai Y, Xia J, Zhou X, Xu S, et al. Risk factors associated with acute respiratory distress syndrome and death in patients with coronavirus disease 2019 pneumonia in Wuhan, China. J Am Med Assoc Intern Med 2020; 180 (7): 934-43.

21. Little $P$, Read RC, Amlot R. Reducing risks from coronavirus transmission in the home-the role of viral load. Br Med J 2020; 369: $\mathrm{m} 1728$. 